\title{
The importance of age and weight on cavopulmonary shunt (stage II) outcomes after the Norwood procedure: Planned versus unplanned surgery
}

David J. Barron, MD, Intisar Ul Haq, MD, Adrian Crucean, MD, John Stickley, BSc, Phil Botha, MD, Natasha Khan, MD, Timothy J. Jones, MD, and William J. Brawn, MD

\section{ABSTRACT}

Objective: The study objective was to evaluate the outcomes of the cavopulmonary shunt after the Norwood procedure with a particular focus on age, weight, and whether surgery was planned or expedited by clinical findings.

Methods: We studied 297 consecutive patients with hypoplastic left heart syndrome undergoing the cavopulmonary shunt operation between 2002 and 2014. All patients underwent the Norwood procedure with a right ventricle to pulmonary artery conduit. Unplanned surgery was any situation in which surgery was expedited because of refractory desaturation, angiographic findings, or failure to discharge.

Results: There were 222 planned and 75 unplanned procedures. Planned surgery was performed at median of 5.4 (4.6-6.3) months compared with 3.9 (3.3-5.0) months for the unplanned group $(P<.001)$. Early mortality was $1.3 \%$ in the planned group compared with $5.3 \%$ in the unplanned group $(P=.07)$. At 5 and 10 years, survival was significantly worse in the unplanned group $(73 \%$ vs $85 \%, P=.03$ ). On multivariable analysis, atrioventricular valve regurgitation, the need for atrioventricular valve repair, and impaired ventricular function (graded composite variable) were the predominant risk factors (hazard ratio [HR], 7.49), with an incremental risk within these 3 variables. The planned versus unplanned nature of the surgery was less significant (HR, 1.75) because it was eclipsed by ventricular function and tricuspid intervention. Additional factors were aortic atresia/mitral atresia subgroup (HR, 2.07) and somatic growth (z-score weight) at the time of surgery (HR, 0.60 for z-score change from -2.54 to -1.16 ). Age and weight at surgery were not significant risk factors for survival. Interstage interventions on the aortic arch or right ventricle to pulmonary artery conduit did not adversely influence outcomes.

Conclusions: The ventricular function, atrioventricular valve regurgitation, and need for tricuspid intervention were the primary risk factors for survival after the cavopulmonary shunt operation. Although unplanned surgery is associated with poor outcomes, this is related to the primary risk factors and not the timing or age of the patient. Somatic growth also has a significant influence on survival. Thus, an earlier cavopulmonary shunt operation is safe in infants who are thriving, but those with poor weight gain are at higher risk. (J Thorac Cardiovasc Surg 2017;154:228-38)

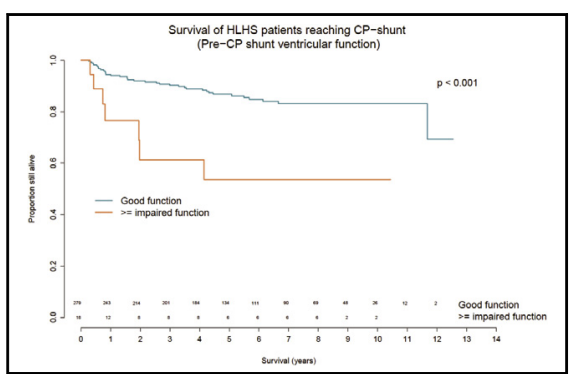

Survival of patients with HLHS reaching CPS (pre-CPS ventricular function).

\section{Central Message}

Early and late outcomes after stage II postNorwood depend on ventricular and tricuspid valve function. Age and weight at surgery are not risk factors in thriving patients (z-scored weight).

\section{Perspective}

The ideal age and weight for stage II postNorwood are not clear, especially if surgery is expedited for clinical concerns. This study showed that ventricular function, AVV regurgitation, and AVV repair were the strongest risk factors for early and late mortality independently of the surgery being planned or unplanned. The age and weight at surgery are not risk factors if patients are thriving ( $\mathrm{z}$-scored weight)

See Editorial Commentary page 239.

See Editorial page 226.
From the Department of Cardiac Surgery, Birmingham Children's Hospital, Birmingham, United Kingdom.

Read at the 95th Annual Meeting of The American Association for Thoracic Surgery, Seattle, Washington, April 25-29, 2015.

Received for publication Feb 19, 2016; revisions received Dec 20, 2016; accepted for publication Dec 30, 2016; available ahead of print Feb 9, 2017.
Address for reprints: Adrian Crucean, MD, Department of Cardiac Surgery, Birmingham Children's Hospital, Steelhouse Lane, Birmingham B4 6NH, United Kingdom (E-mail: adrian.crucean@bch.nhs.uk).

0022-5223/\$36.00

Copyright (C) 2017 by The American Association for Thoracic Surgery

http://dx.doi.org/10.1016/j.jtcvs.2016.12.036 

Abbreviations and Acronyms
AAMA $=$ aortic atresia/mitral atresia
AVSD $=$ atrioventricular septal defect
$\mathrm{AVV}=$ atrioventricular valve
CPS = cavopulmonary shunt
HLHS = hypoplastic left heart syndrome
HR = hazard ratio
ICU = intensive care unit
$\mathrm{IQR}=$ interquartile range
$\mathrm{RV}-\mathrm{PA}=$ right ventricle to pulmonary artery

Scanning this QR code will take you to the article title page.

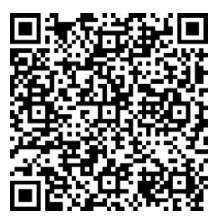

The optimum timing for cavopulmonary shunt (CPS) stage II palliation after the Norwood procedure is not fully defined, generally being performed at an age of 4 to 6 months when pulmonary vasculature has matured and pulmonary vascular resistance has decreased to a level at which a venous shunt can be well tolerated. ${ }^{1-3}$ However, concerns over the relative fragility of the Norwood circulation and interstage mortality/morbidity have led to a general trend to earlier rather than later stage II surgery to achieve a more secure hemodynamic situation. ${ }^{4,5}$

Conversion to stage II removes the volume load from the circulation and excludes the risks of a shunt-dependent circulation, such as shunt thrombosis, infection, stenosis, or sudden death. Although the use of the right ventricle to pulmonary artery (RV-PA) conduit may have reduced the latter risk, there remains significant attrition of patients during the interstage period in most published series, ${ }^{1,3,6,7}$ sustaining the interest in converting to cavopulmonary circulation as soon as possible. Stage II surgery also provides an opportunity to address any residual lesions, such as branch pulmonary artery stenosis, atrioventricular valve (AVV) regurgitation, or residual atrial septal tissue and so further optimize the hemodynamics. ${ }^{8}$

The drawbacks of performing the stage II surgery too early are concerns that the pulmonary vasculature may not have fully developed, resulting in a relatively high pulmonary vascular resistance that may lead to hypoxia, venous hypertension, and prolonged pleural effusions with extended in-hospital stay and a higher early mortality.

Ideally, all stage II surgery would be planned to be undertaken at a certain age or when a combination of age and weight targets have been reached. However, procedures cannot always be planned, and changes in the clinical status of the infant, most commonly persistently low oxygen saturations, poor clinical progress, or the development of significant hemodynamic problems (eg, AVV regurgitation or restrictive atrial septum), require the procedure to be expedited.

This study examines the impact of age and timing of CPS surgery with particular focus on whether the procedure was planned (elective) or unplanned (nonelective). A range of morphologic, procedural, and physiologic variables were included to analyze the impact of these factors on outcome, particularly with relevance to how these influenced timing of the CPS. The study also examined whether the need for interstage reinterventions influenced outcomes and whether z-scored weight (ie, age-appropriate growth) was more important than absolute weight at the time of CPS. The aim was to create a risk model to help in decision making and understand the relative impact of key morbidities on the outcomes of stage II surgery.

\section{MATERIALS AND METHODS}

All patients undergoing CPS after the Norwood procedure for hypoplastic left heart syndrome (HLHS) between 2002 and 2014 were included in the study. All procedures were performed in a single institution (Birmingham Children's Hospital, Birmingham, UK). The year 2002 was chosen because this was the point at which an institutional decision was taken to change from a modified Blalock-Taussig shunt to an RV-PA conduit. Thus, all patients had undergone the Norwood procedure using a similar technique of RV-PA conduit.

All patients had HLHS including variants with unbalanced atrioventricular septal defect (AVSD). Patients with left ventricular dominant morphology, such as double-inlet left ventricle with subaortic, annular, or arch obstruction, were excluded from the study. The study was approved by the local ethical committee, and parental consent was waived because this was a retrospective study using existing available data.

Patient records and electronic cardiac database (Heartsuite, Systeria, Glasgow, Scotland) were reviewed for all cases. Variables studied included age and weight at the time of CPS, ventricular function, morphologic subtype (divided into aortic atresia/mitral atresia [AAMA], aortic atresia/mitral stenosis, aortic stenosis/mitral stenosis, and unbalanced AVSD), number and timing of reinterventions, AVV regurgitation, AVV reoperation, ventricular function, bypass data at both stage I and II, and hospital and intensive care unit (ICU) stay. The end point was transplant-free survival at the time of last follow-up. Ventricular function was based on echocardiographic assessment at the time of CPS, and moderate or greater impairment was defined as "impaired function" in the analysis in line with previous studies. ${ }^{5,9}$ AVV regurgitation also was based on echocardiographic assessment and graded as trivial, mild, moderate, or severe. Any moderate or greater regurgitation was deemed significant and as an indication for valve repair. All assessments of ventricular function and AVV regurgitation were performed by the same investigator. Mechanical support (extracorporeal membrane oxygenation) was used in only 2 patients in the series and so was not considered in the model.

Planned management is to perform routine angiography at 3 to 4 months of age with the target age for CPS at 5 to 6 months. A planned case was defined as an operation for a patient admitted from home while being on the surgical waiting list. Unplanned procedures encompassed all other cases and included patients admitted for refractory desaturation or clinical deterioration, patients with prolonged inpatient stay who were not fit to be discharged, and patients admitted for cardiac angiography who became unstable after catheterization or in whom significant lesions were found 
TABLE 1. Patient characteristics, counts with percentage or median with interquartile range

\begin{tabular}{|c|c|c|c|c|}
\hline Variable & All patients & Planned & Unplanned & $P$ value \\
\hline Patients & 297 & 222 & 75 & \\
\hline Morphology & & & & .194 \\
\hline AAMA & $103(34.7 \%)$ & $77(34.7 \%)$ & $26(34.7 \%)$ & \\
\hline AAMS & $57(19.2 \%)$ & $47(21.2 \%)$ & $10(13.3 \%)$ & \\
\hline ASMS & $87(29.3 \%)$ & $58(26.1 \%)$ & $29(38.7 \%)$ & \\
\hline Shone syndrome & $10(3.4 \%)$ & $9(4.1 \%)$ & $1(1.3 \%)$ & \\
\hline UAVSD & $25(8.4 \%)$ & $21(9.5 \%)$ & $4(5.3 \%)$ & \\
\hline Other & $15(5.1 \%)$ & $10(4.5 \%)$ & $5(6.7 \%)$ & \\
\hline \multicolumn{5}{|l|}{ Demographics } \\
\hline Age (d) & $155(125-182)$ & $166(139-194)$ & $118(102-152)$ & $<.001$ \\
\hline Weight (kg) & $6.0(5.3-6.7)$ & $6.2(5.6-6.9)$ & $5.2(4.7-6.0)$ & $<.001$ \\
\hline Weight $z$ score & $-1.9(-2.5$ to -1.2$)$ & $-1.8(-2.4$ to -1.0$)$ & $-2.1(-2.9$ to -1.5$)$ & .021 \\
\hline \multicolumn{5}{|l|}{ Operative details } \\
\hline Bypass time (min) & $52(46-60)$ & $52(46-59)$ & $56(48-64)$ & .21 \\
\hline Size of initial RV-PA conduit & $5.0(5.0-5.0)$ & $5.0(5.0-5.0)$ & $5.0(5.0-5.0)$ & .145 \\
\hline Interstage interventions & 166 & 129 & 37 & .178 \\
\hline \multicolumn{5}{|l|}{ Postoperative details } \\
\hline ICU length of stay (h) & $26.0(22.7-68.2)$ & $25.1(22.2-43.4)$ & $55.7(25.7-117.8)$ & $<.001$ \\
\hline Ward length of stay (d) & $8(6-13)$ & $7(6-11)$ & $9(6-17)$ & .009 \\
\hline
\end{tabular}

$\overline{A A M A}$, Aortic atresia/mitral atresia; $A A M S$, aortic atresia mitral stenosis; $A S M S$, aortic stenosis mitral stenosis; $U A V S D$, unbalanced atrioventricular septal defect; $R V-P A$, right ventricle to pulmonary artery; $I C U$, intensive care unit.

that were thought to require inpatient management. Other reasons were signs of infection or thrombosis of the shunt that were not responding to conservative treatment.

\section{Surgical Technique}

All patients had undergone an initial Norwood procedure using an RV-PA conduit. A 5-mm shunt was used for patients weighing more than $2.5 \mathrm{~kg}$ and a $4-\mathrm{mm}$ shunt for those weighing $2.5 \mathrm{~kg}$ or less. The techniques for Norwood have been described..$^{10}$ Bidirectional CPS was performed in a standard fashion for the entire patient group. Procedures were performed on hypothermic cardiopulmonary bypass under a short period of circulatory arrest at $18^{\circ} \mathrm{C}$ for the cavopulmonary anastomosis, using absorbable sutures (polydioxanone sodium). Any narrowing in the central pulmonary arteries was addressed at the same procedure using a pulmonary homograft patch. The proximal stump of the RV-PA conduit was oversewn and left attached to the right ventricle. Details of any other additional procedures are outlined in the "Results" section.

\section{Statistical Methods}

Exploratory analyses incorporating graphical and tabular displays assessed evidence in favor of trends and associations. Skewed data are presented as median and interquartile range (IQR). Categoric data are expressed as counts and percentages when appropriate. Categoric variables were analyzed using the Fisher exact test, and continuous variables were assessed using the Wilcoxon rank-sum test.

Overall survival was estimated using the Kaplan-Meier method and was measured from the date of CPS to transplantation or death due to any cause. The log-rank test was used to compare survival between subgroups.

Cox proportional hazard analysis was performed using a stepwise selection method aiming to identify a parsimonious model. Explanatory variables were removed when they did not contribute to the model fit using the Akaike information criterion, and the model resulting in the lowest Akaike information criterion was selected. Checks were carried out to support the proportional hazard assumptions. Continuous variables entered the model as such and were presented graphically as quintiles.

All statistical analyses were performed by using R version 3.2.2 (201508-14). The packages survival, rms, and ggplot2 along with packages in the base version were used for presenting data and for the analysis. Significance testing was 2 sided.

\section{RESULTS}

During the study period, 297 patients underwent CPS. The patient demographics, morphologic subtype, operative details, and postoperative length of stay are summarized in Table 1. Within this patient group, the median weight at time of Norwood was $3.1 \mathrm{~kg}$ (IQR, 2.8-3.5 kg), which is a z-score weight of -1.1 ( -1.9 to -0.3 ). Median follow-up was 4.9 years (IQR, $2.0-8.0$ years), and follow-up was $100 \%$ complete.

Early mortality occurred in 7 cases $(2.3 \%)$. The median age at CPS in the entire cohort was 5.1 months (IQR, 4.1-5.9 months), and the median weight was $6.0 \mathrm{~kg}$ (IQR, $5.3-6.7 \mathrm{~kg}$ ). There were 23 late deaths in the entire series with a 5- and 10-year survivals of $85 \%$ and $82 \%$, respectively, and a further 4 patients have undergone heart transplantation. Of the remaining patients, 128 have undergone stage III surgery, total cavopulmonary connection, or Fontan completion, and the remaining patients are awaiting stage III surgery.

A total of 28 AVV repairs were performed: 19 at the time of CPS, of which 12 were in the planned and 7 were in the unplanned group (5.4\% vs 9.3\%); 3 patients before the CPS ( 1 in the planned and 2 in the unplanned group); and 7 patients had the AVV repaired at stage I ( 4 in the planned and 3 in the unplanned group). One patient underwent AVV repair at stage I, during the interstage phase and at the CPS.

\section{Planned Versus Unplanned Surgery}

Planned (elective) surgery was performed in 222 cases, with unplanned CPS being performed in 75 cases. The early 


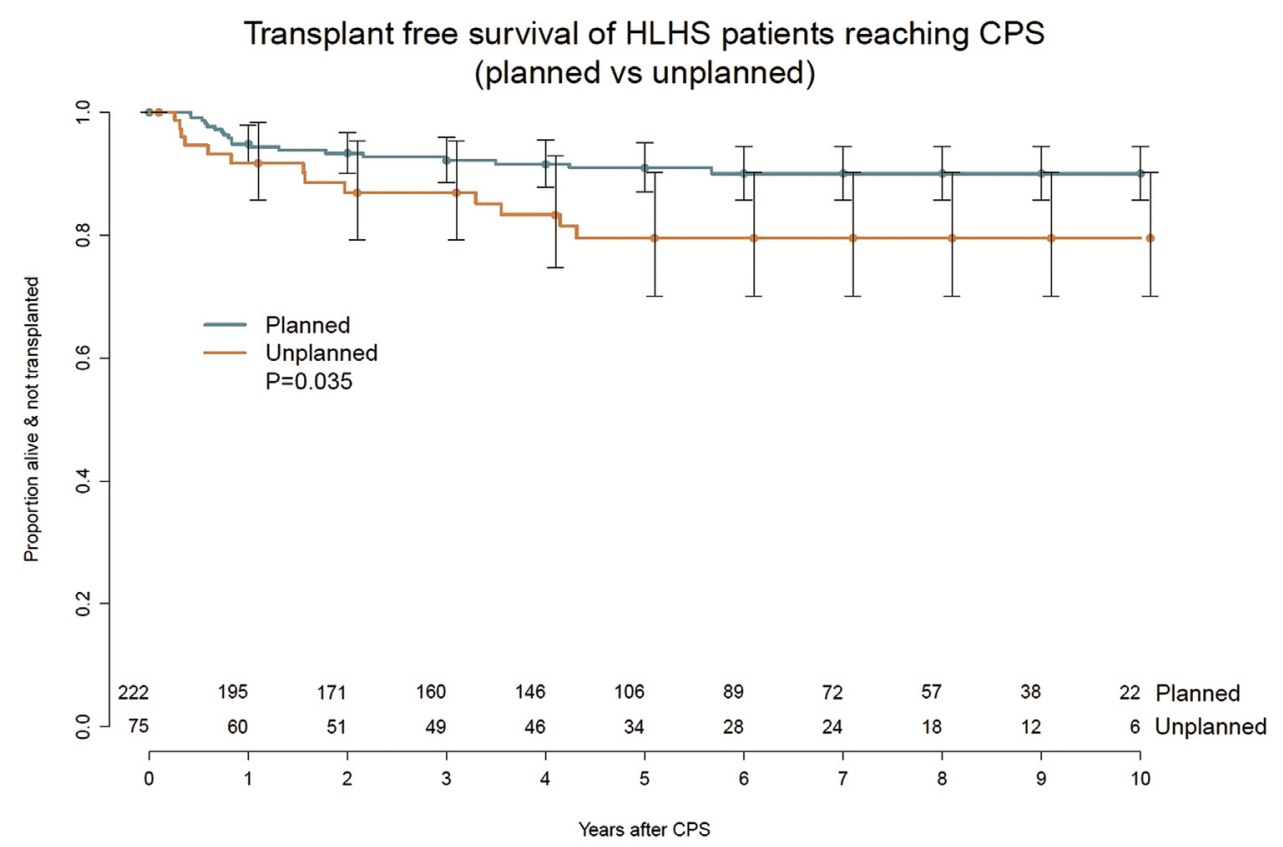

FIGURE 1. Survival of patients with HLHS reaching CPS (planned vs unplanned patients). HLHS, Hypoplastic left heart syndrome; CPS, cavopulmonary shunt.

mortality was $1.3 \%(3 / 222)$ in the planned group compared with $5.3 \%(4 / 75)$ in the unplanned group $(P=.07)$. Survival of the planned versus unplanned cases is shown in Figure 1, and patient characteristics are shown in Table 1. There was significantly better survival in the planned group; 5- and 10-year survivals were $88 \%$ versus $75 \%$ at 5 years and $85 \%$ versus $73 \%$ at 10 years. The median age at CPS for the planned group was 5.5 months (4.6-6.5) compared with 3.9 months (3.4-5.1) in the unplanned group $(P<.001)$. Correspondingly, the weight at CPS was $6.2 \mathrm{~kg}$ (IQR, 5.6-6.9 $\mathrm{kg}$ ) for the planned group compared with $5.2 \mathrm{~kg}$ (IQR, 4.7-6.0 kg) in the unplanned group $(P<.001)$. There was no difference in the presence of morphologic subtypes between the groups (Figure 2). In terms of operative and perioperative data, there was no difference in bypass times between the 2 groups, but the ICU length of stay (median 25 vs 56 hours) and hospital length of stay (median 7 vs 9 days) were significantly longer in the unplanned group $(P<.01)$, as shown in Table 1. There were 5 patients who remained in the hospital between stage I and II, all of whom were (by definition) part of the unplanned group; failure to discharge was not an independent risk factor for poor outcome.

\section{Morphologic Subtypes and Reinterventions}

Analysis of morphologic subtypes across the entire cohort showed that the AAMA subgroup was associated with decreased survival compared with all other groups, with a 10 -year survival of $76 \%$ versus $85 \%(P=.04$, Figure 3). Neither the unbalanced AVSD group nor the aortic atresia/mitral stenosis group was identified as a risk factor in this analysis. The need for aortic arch intervention, including those performed at planned prestage II angiography or as an additional procedure because of clinical or echocardiography findings, was not associated with any difference in outcome after CPS (Figure 4, A). Likewise, the need for reinterventions on the RV-PA conduit did not influence the outcomes (Figure 4, B). Thus, interstage interventions on both the aortic arch and the RV-PA conduit restored the patient's medical trajectory to expected outcomes. However, the need for AVV repair as a separate procedure or at the time of CPS was strongly associated with decreased survival in all patient groups (Figure 5). This was the strongest single procedural or morphologic risk factor for death or transplantation, with survival at 5 years of $50 \%$ compared with $88 \%$ in the group that required no $\mathrm{AVV}$ intervention $(P<.001)$. The second strongest single risk factor was impaired ventricular function at the time of the CPS as defined at echocardiography and angiography (Figure 6). Survival at 5 years was $87 \%$ for those with good or mildly impaired function compared with $54 \%$ for those with moderate or greater impairment of function $(P<.001)$.

\section{Relationship of Age, Weight, and Standardized Weight}

Because age and weight are correlated variables, the analysis looked at both factors independently but also by indexing weight for age (using z-scores) to reflect somatic growth relative to age. The population was broken down 


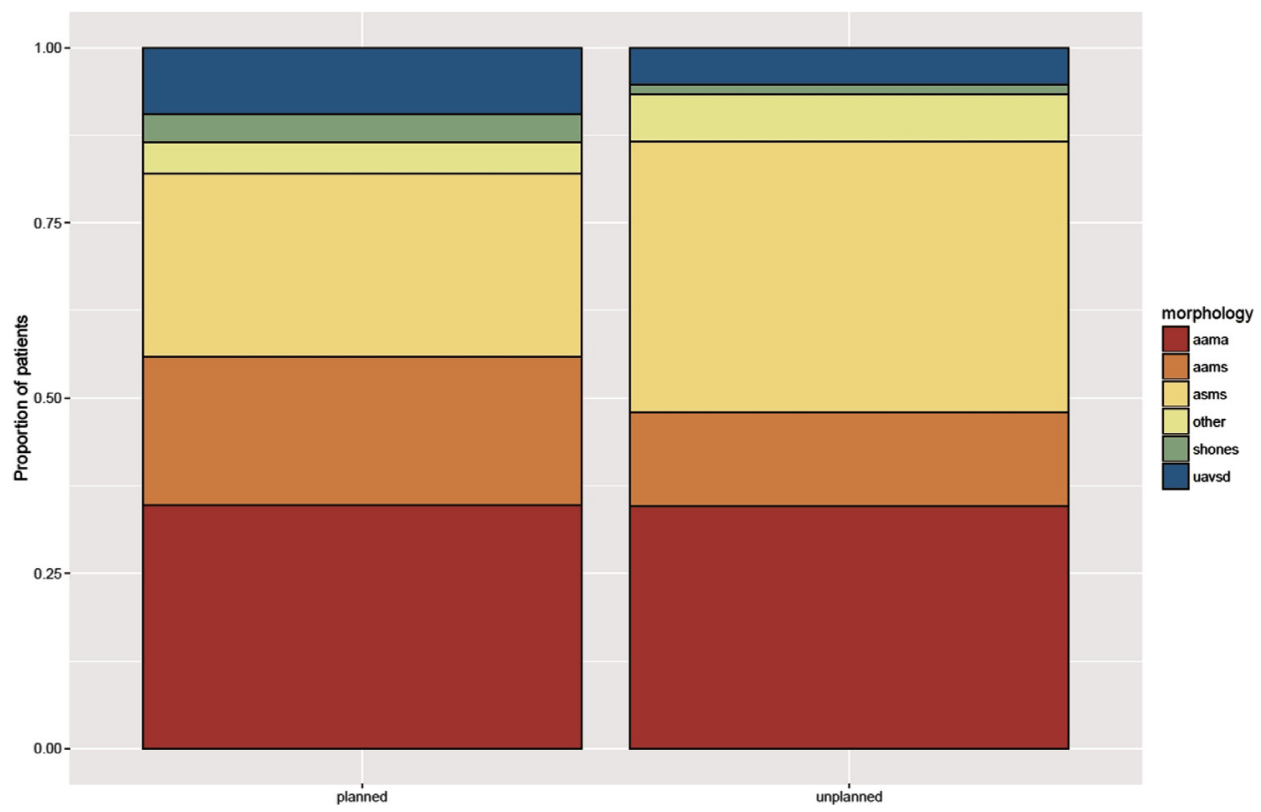

FIGURE 2. Morphology of patients reaching CPS. CPS, Cavopulmonary shunt; aama, aortic atresia/mitral atresia; aams, aortic atresia mitral stenosis; asms, aortic stenosis mitral stenosis; uavsd, unbalanced atrioventricular septal defect.

into quintiles according to age and weight at the time of CPS for the purposes of displaying the data, with survival shown in Figure 7, $A$ and $B$. Univariable analysis showed that age at CPS was not an independent risk factor for outcome. However, z-scored weight at the time of CPS was a significant risk factor $(P=.03)$ with 5-year survival of lowest versus highest quintile of z-score weight of $74 \%$ versus $95 \%$.

\section{Creation of a Risk Model}

A Cox proportional hazard analysis was performed using all studied variables (listed in Table 2) to ascertain the factors that influenced survival. Table 2 and Figure 8 show the significant risk factors for survival after CPS. Impaired ventricular function, moderate or greater AVV, and AVV repair were closely inter-related as significant risk factors. Consequently, using them as individual risk

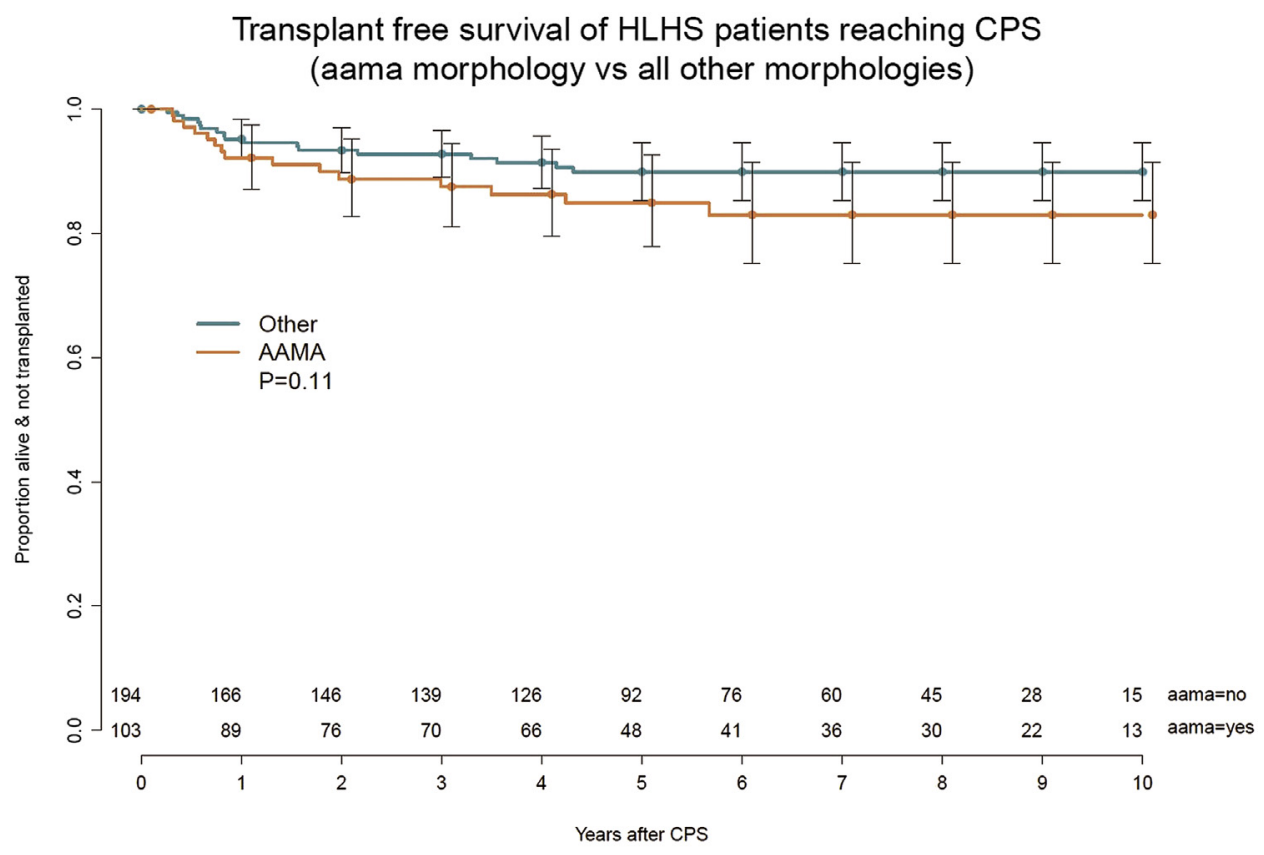

FIGURE 3. Survival of patients with HLHS reaching CPS (AAMA morphology vs other morphologies). HLHS, Hypoplastic left heart syndrome; CPS, cavopulmonary shunt; AAMA, aortic atresia/mitral atresia. 


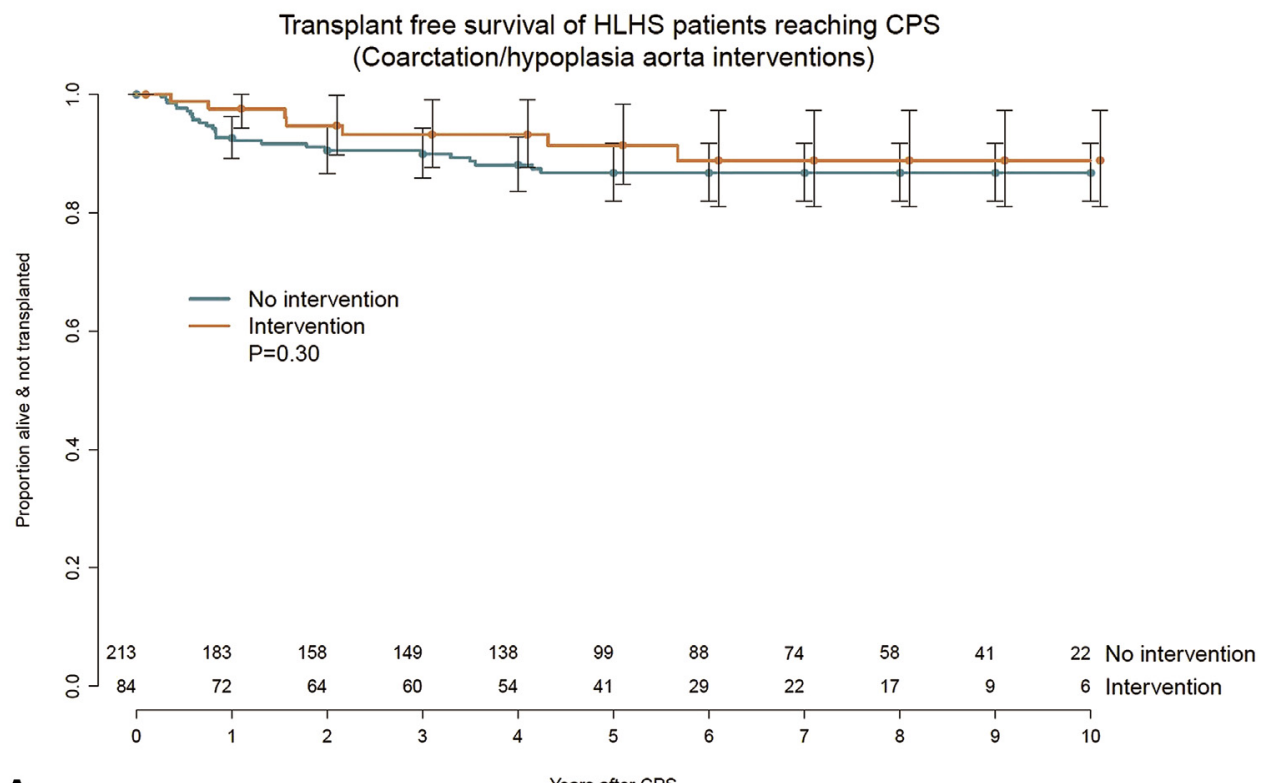

A

Transplant free survival of HLHS patients reaching CPS (RV-PA conduit interventions)

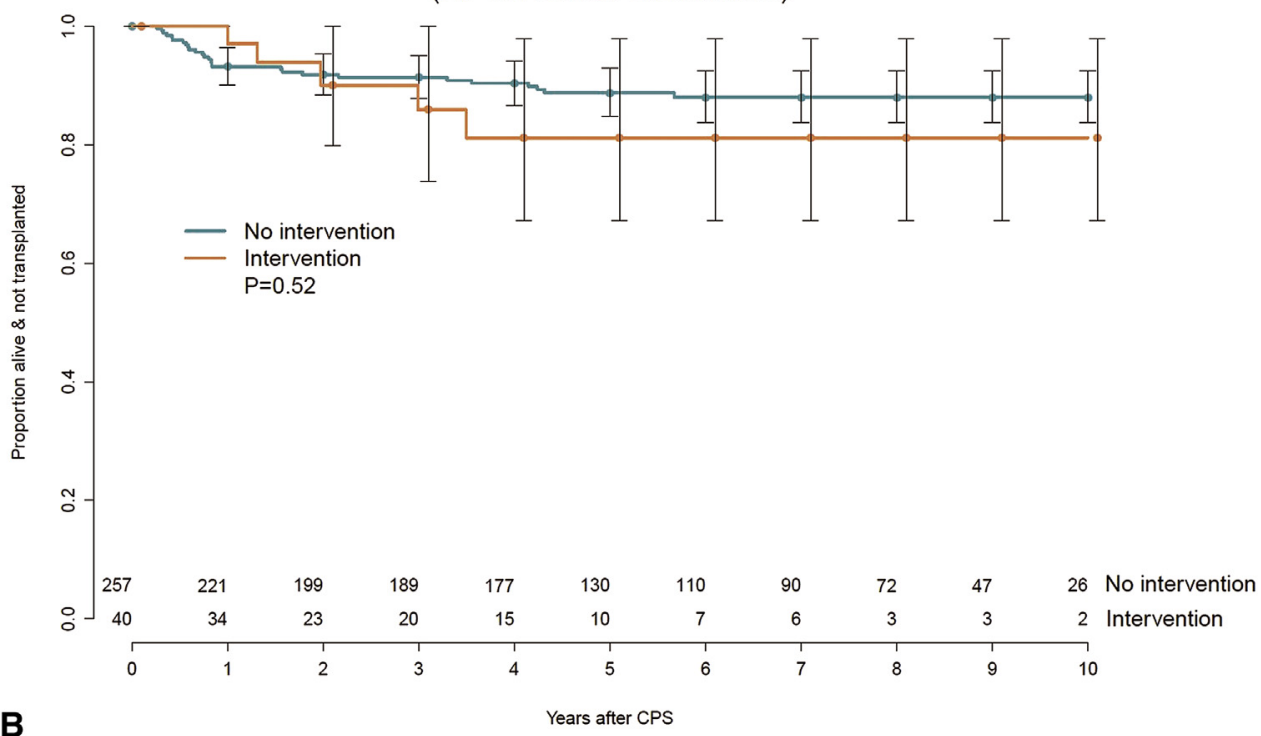

FIGURE 4. A, Survival of patients with HLHS reaching CPS (coarctation/hypoplasia aorta interventions). B, Survival of patients with HLHS reaching CPS (RV-PA conduit interventions). HLHS, Hypoplastic left heart syndrome; $C P S$, cavopulmonary shunt; $R V$-PA, right ventricle to pulmonary artery.

factors confounded the modeling, and the best fit was found using all 3 as a graded composite risk factor (hazard ratio [HR], 7.49). Within this model, each of these 3 factors had an incremental influence on long-term outcome (Figure 8). Additional significant risk factors for survival were the $z$-scored weight at the time of CPS and the AAMA subgroup. On multivariable analysis, the fact that surgery was planned or unplanned still carries a small increased hazard (HR, 1.75), but this becomes far less significant in comparison with the influence of impaired ventricular function and AVV regurgitation. Likewise, the age and absolute weight at time of CPS were not identified as risk factors, as discussed earlier.

\section{DISCUSSION}

Surgery for HLHS remains one of the greatest challenges in congenital heart disease, and understanding ways of improving outcomes has been logically focused on the neonatal management, where the majority of risk and mortality lies. However, there has been increasing recognition 


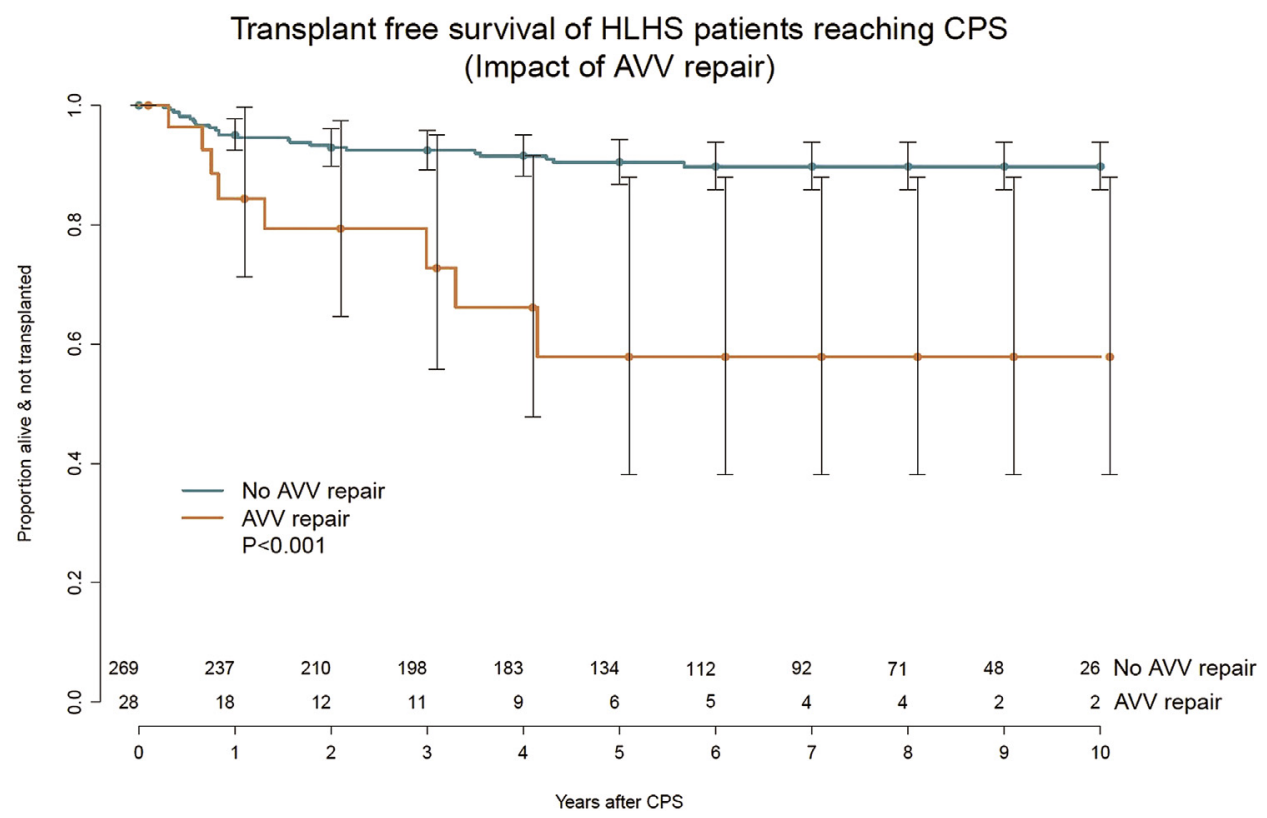

FIGURE 5. Survival of patients with HLHS reaching CPS (impact of AVV repair). HLHS, Hypoplastic left heart syndrome; CPS, cavopulmonary shunt; $A V V$, atrioventricular valve.

over the past 15 years of the risks and attrition of interstage mortality between stage I and II, with progress being made in trying to mitigate the risks, particularly in the field of interstage surveillance to preempt clinical deterioration. ${ }^{8,11,12}$

Mortality after the stage II procedure has a marked stepchange reduction, ${ }^{1}$ and the survival after hospital discharge from stage II is extremely good with a low risk of sudden death and a survival of $88 \%$ to $93 \%$ at 6 to 10 years. $^{3,13}$
Therefore, there is considerable focus on successfully negotiating stage II with the knowledge that this provides the patients with a more robust circulation, removing the volume load of the systemic shunt and excluding the shunt-related risks of thrombosis, stenosis, outgrowth, and sudden death. Consequently, the timing of the stage II surgery has been brought forward to as early as possible, but the question remains as to the correct time, when it is too early, and the risks in planning the timing of the stage II procedure.

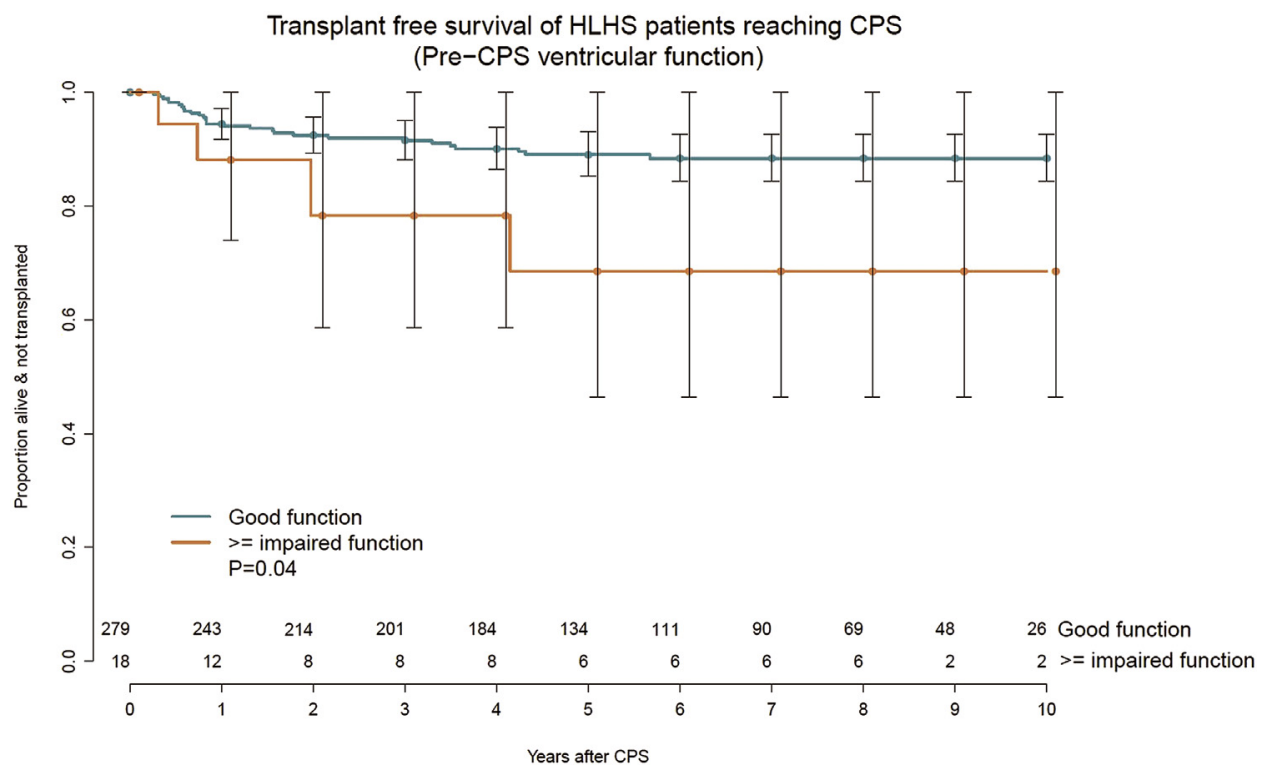

FIGURE 6. Survival of patients with HLHS reaching CPS (pre-CPS ventricular function). HLHS, Hypoplastic left heart syndrome; CPS, cavopulmonary shunt. 


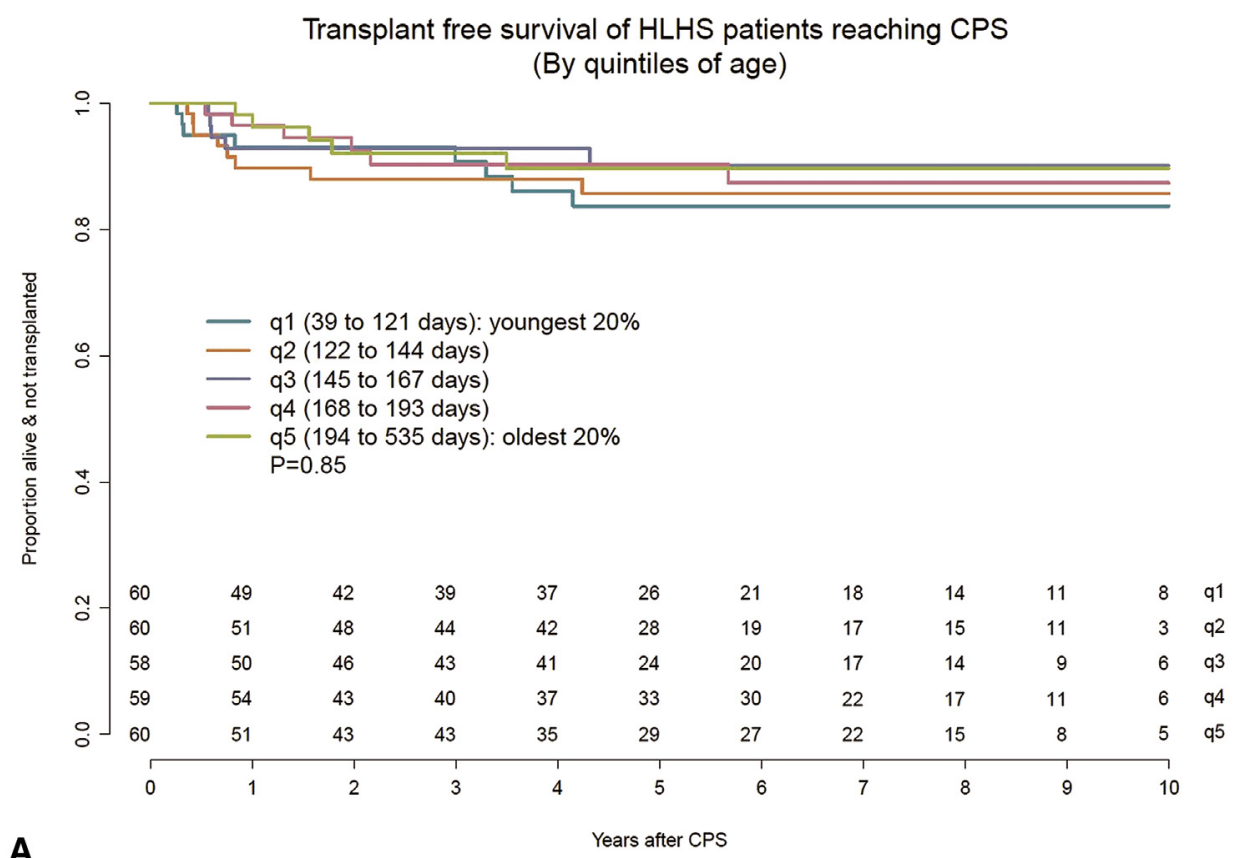

A

Transplant free survival of HLHS patients reaching CPS

(By quintiles of $z$-score weight)

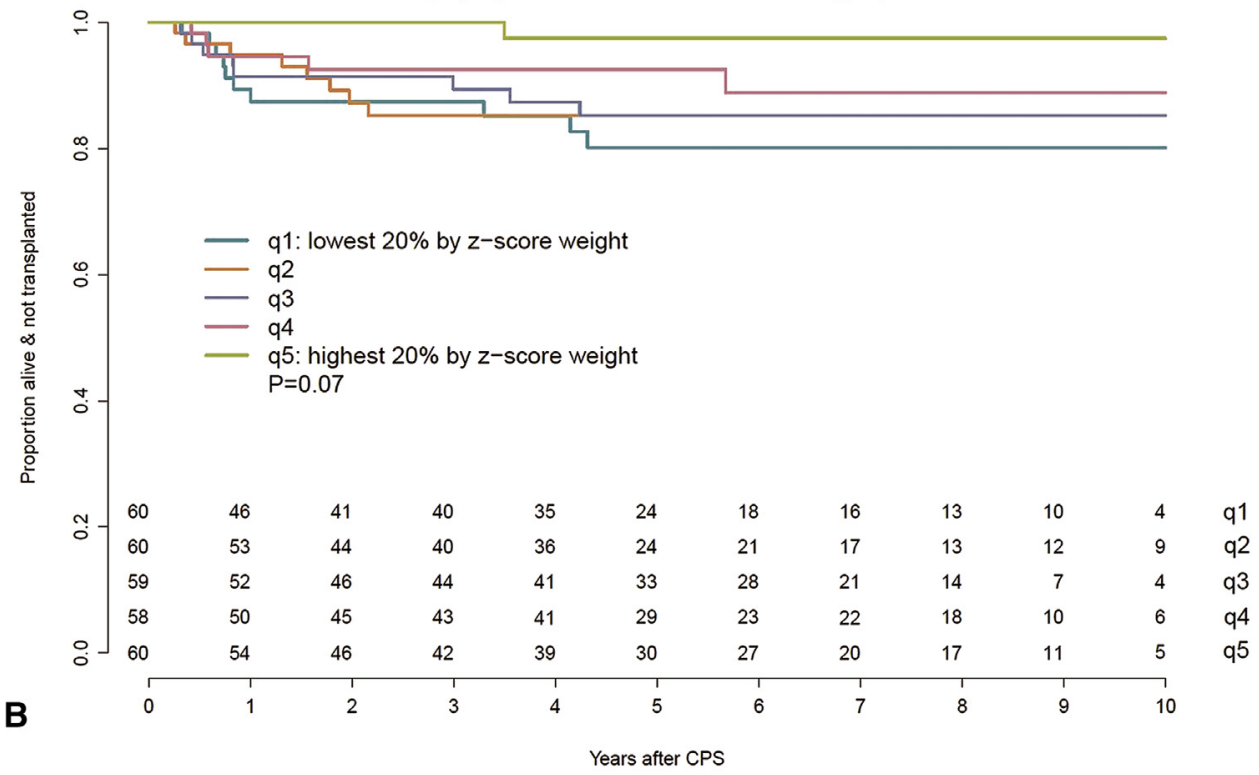

FIGURE 7. A, Survival of patients with HLHS reaching CPS (by quintiles of age). B, Survival of patients with HLHS reaching CPS (by quintiles of z-score weight). HLHS, Hypoplastic left heart syndrome; CPS, cavopulmonary shunt.

This study has set out to answer some of these questions by examining a large cohort of consecutive patients and analyzing the outcomes according to the age, weight, associated lesions, and timing of the CPS surgery.

Early mortality for the entire cohort was low $(2.3 \%)$, which makes it difficult to use early death as a useful end point for understanding the impact of the potential risk factors. This outcome compares favorably with the recent publication on the single ventricle reconstruction trial with an overall early mortality of $4.3 \%{ }^{14}$ The survival at 5 and 10 years is more useful, and the study has shown that moderate or greater AVV regurgitation, AVV reintervention, and impaired ventricular function are the strongest single risk factors for poor late outcome, with an HR of 7.49. Tricuspid or AVV regurgitation repeatedly has been shown to be a strong determinant of outcome in HLHS 
TABLE 2. Cox proportional hazard model coefficients and hazard ratios (with $95 \%$ confidence interval) with examples for $\mathrm{z}$-scored weight (Figure 8)

\begin{tabular}{lll}
\hline \multicolumn{1}{c}{ Variable } & \multicolumn{1}{c}{ HR } & Selected \\
\hline AAMA morphology (yes:no) & $2.07(1.02-4.19)$ & Yes \\
z-score weight (change from & $0.61(0.39-0.92)$ & Yes \\
$\quad-2.54$ to -1.16$)$ & & \\
Planned (yes:no) & $1.74(0.84-3.64)$ & Yes \\
Composite variable (AVV repair, & & \\
$\quad$ AVV regurgitation, ventricular function) & $1.13(0.36-3.49)$ & Yes \\
1 (only 1 present) & $5.05(1.88-13.60)$ & Yes \\
2 (any 2 factors present) & $7.49(1.65-33.97)$ & Yes \\
3 (all 3 present) & & No \\
Weight at CPS & & No \\
Age at CPS & & No \\
Bypass time (using restrictive cubic spline) & & No \\
RV-PA intervention interstage (I-II) & & No \\
Coarctation intervention interstage (I-II) & &
\end{tabular}

$H R$, Hazard ratio; $A A M A$, aortic atresia/mitral atresia; $A V V$, atrioventricular valve; $C P S$, cavopulmonary shunt; $R V-P A$, right ventricle to pulmonary artery.

and is further supported by these studies findings. ${ }^{1,3,4,13-15}$ However, in contrast to many of these studies, unbalanced AVSD was not specifically a risk factor in this series. This may reflect that the major hazard for unbalanced AVSD occurs at the time of the Norwood or prestage II and that those surviving post-CPS are those with less AVV regurgitation, further improved by the more favorable volume loading conditions after stage II. These findings suggest that the severity of AVV regurgitation per se is more important than the underlying morphology of the valve in the post-CPS follow-up.

The AAMA subgroup was shown to be an independent risk factor for death, although not as strongly as the AVV function. The impact of the various morphologies within HLHS has been extensively examined in the past, and few studies have identified any single group consistently being

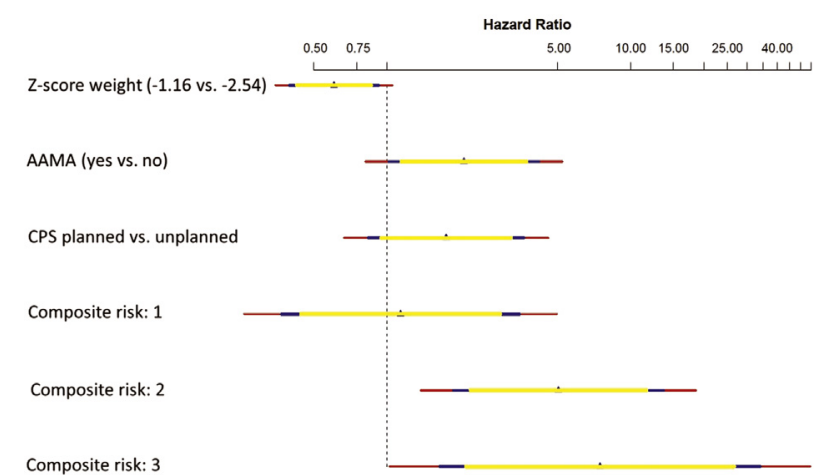

FIGURE 8. Hazard ratio plot with $90 \%, 95 \%$, and $99 \%$ confidence interval for variables included in the final Cox model. CPS z-score is the $\mathrm{z}$-scored weight at time of CPS. The hazard ratio for CPS z-score is for quartile 1 versus quartile 3. AAMA, Aortic atresia/mitral atresia; CPS, cavopulmonary shunt. at greater risk. A small-sized ascending aorta (generally regarded as $\leq 2 \mathrm{~mm}$ ) is strongly linked to aortic atresia and frequently has been shown to be a risk factor in Norwood surgery. ${ }^{2,3,16,17}$ This reflects the technical difficulty in ensuring adequate coronary blood flow after the surgical reconstruction of the aortic arch when dealing with such a small aorta. Some of the risk associated with aortic atresia seems to have been mitigated through the use of the RVPA conduit, probably through maintaining better diastolic coronary perfusion. ${ }^{18,19}$ The findings of this study suggest a sustained risk in the aortic atresia subgroup that continues beyond stage II surgery. This is an important observation because many studies focus on the risk of aortic atresia in the setting of neonatal outcomes, but not beyond that age. This could be due to the continuing impact of suboptimal coronary flow during the first months of life or may reflect ongoing compromise of coronary perfusion leading to chronic myocardial dysfunction in this group.

Catheter reinterventions on the aortic arch and RV-PA conduit are well documented as the most common interstage procedures between the Norwood and stage II. ${ }^{3,9,20}$ This study showed that patients undergoing interventions on the aorta and the RV-PA conduit had no increased risk on early or late survival after CPS. This is an encouraging finding and would suggest that these interventions are successful in restoring these patients to a normal trajectory in terms of outcome at CPS, a finding that was also borne out in the single ventricle trial. ${ }^{20}$

The target age for stage II in our institution has been 4 to 6 months, which is a typical age range in respect of most large published series around the world (including the North American Single Ventricle Reconstruction trial, in which the mean age was 5.4 months ${ }^{14}$ ). One of the key findings of this study was that age at CPS, when considered as an independent variable, was not a risk factor for mortality. The survival curves for age by quintile showed similar outcomes (Figure 7, A). At the time of CPS, both weight and z-scored weight show a difference in outcome when compared by quintile; however, in the Cox model, z-scored weight was retained. Nevertheless, it is difficult to separate age from weight because they are so interdependent in this age group of patients. When weight was z-scored for age (reflecting somatic growth and "failure to thrive"), it became a consistent independent risk factor for mortality. There has been increasing evidence that earlier CPS can be performed safely, but with the concern that it can be associated with greater morbidity and longer hospital stay. ${ }^{5,21}$ However, this study suggests that these findings may be more related to the unplanned nature of some of the earlier CPS procedures (and, in turn, their associated risk factors) and that, so long as the patient is thriving with good ventricular and AVV function, then earlier CPS can have excellent outcomes. 
Somatic weight gain and weight for age are wellrecognized, albeit nonspecific markers of well-being in children, and their prognostic value is clearly multifactorial. Nevertheless, the importance of $\mathrm{z}$-scored weight in this context can be a valuable observation to be considered in these patients, ${ }^{13,22,23}$ mirroring the recognition that weight gain forms such a strong predictive factor in the interstage surveillance of these infants.

Thus, in terms of age, these findings would suggest that early CPS carries no increased risk as long as somatic weight gain is normal. However, in infants who are failing to thrive, the risk incrementally increases with the degree of deviation from expected weight.

Elective planning for CPS is the ideal setting, but there is a significant group of patients in whom the timing of the surgery is not planned. Clinical deterioration or unforeseen events supervene, and a decision has to be made whether to expedite the timing of stage II. Typically, this is the patient who presents with persistently low arterial oxygen saturations (refractory to conventional treatment) or patients who are failing to progress with signs of congestive heart failure or simply never stable enough to be discharged. More rarely, the cause is infection or thromboembolic problems with the shunt, requiring urgent reintervention. This group also includes some patients who are clinically more stable but in whom angiography or echocardiography has revealed important anatomic problems that warrant inpatient management without delay.

This study sought to investigate this concept of planned versus unplanned CPS to reflect the clinical scenarios that we are faced with. Approximately one quarter of the cases in this study were in the unplanned group, with the expected findings that this cohort was younger (median age, 3.9 months, 1.5 months younger than the planned group) and of lower weight (median $5.2 \mathrm{~kg}$ vs $6.2 \mathrm{~kg}$ in the planned group) at the time of CPS. Early mortality was higher in the unplanned group, and the ICU and inpatient lengths of stay were longer. During follow-up, it also became clear that the survival of the unplanned group was significantly worse than in the planned group at both 5 and 10 years. This finding was not unexpected, given that the unplanned cases inherently had suboptimal hemodynamics and were clinically less stable, but it is important to recognize the relative contribution of simply being "unplanned" in terms of impact on this patient group. The multivariate model showed that the major risk in these patients is accounted for by the associated risk factors of impaired ventricular function, AVV regurgitation, and AVV repair, but there remains an inherent risk in simply being an unplanned procedure, independent of these factors (HR, 1.74). In itself, this is a valuable observation but needs to be placed in perspective of the other comorbidities that influence survival, and it raises the question whether an intervention can be used to convert an unplanned into a planned procedure just as introduction of more focused nutrition and feeding regimens may be able to improve the $\mathrm{z}$-scored weight and so reduce operative risk. Should these be possible, then it may secure better outcomes at both early and long term. The converse also is true in that a thriving patient with good ventricular function could safely undergo stage II surgery at 4 months and so transition out of the interstage period sooner.

The Cox proportional hazard model was used to study a wide variety of variables to identify the risk factors for outcome after CPS and to describe the HR of each. The highest grade composite risk factor of moderate or greater AVV regurgitation, AVV repair, and preoperative impaired ventricular function was the greatest hazard, with each of these 3 factors carrying an incremental risk to long-term survival. In addition, AAMA subtype and z-score weight at the time of CPS remained independent risk factors for long-term survival. The planned versus unplanned nature of the surgery became less important in this multivariable model, suggesting that the majority of the decreased survival in the unplanned group is accounted for by the prevalence of impaired ventricular function and AVV regurgitation, which were very much the key factors in accounting for the poorer outcomes in the unplanned group. The single ventricle reconstruction trial also described the increased length of stay associated with the unplanned group and noted the increased mortality on univariable analysis; however, it is important to recognize that with multivariable analysis, the influence of the unplanned nature of the surgery (HR, 1.75) is mainly eclipsed as a risk factor when ventricular function, AVV regurgitation, AVV repair, and somatic weight gain are taken into account. ${ }^{14}$

The Cox model can be used to identify patient groups at a higher risk and may assist in planning future management; for example, it may be possible to devise pathways to improve nutrition and weight gain that may be beneficial if surgery can be safely delayed in certain patient subgroups.

\section{Study Limitations}

Because the study examines only those patients undergoing CPS, the risk factors for death after stage I or in the interstage period do not feature. This would underestimate the relative risk of poor ventricular function and severe AVV regurgitation in the entire population. However, as long as this study is recognized as being exclusively focused on those patients who survive to undergo CPS, then the findings remain valid. The study also does not investigate whether patients may have required subsequent interventions on the AVV or the arch after stage II. It would be interesting to know which patient groups may have gone on to require subsequent intervention, but this was beyond the scope of this study. The variables chosen for the proportional hazard model were not exhaustive and were limited 
by practical constraints of available data. Thus, some anatomic details at the time of stage I Norwood and comorbidities after stage I did not feature in the model.

\section{CONCLUSIONS}

Stage II surgery for HLHS carries a low early mortality, but the composite variable of AVV repair, moderate or greater AVV regurgitation, and preoperative ventricular function is the strongest single risk factor for mortality. Failure to thrive (z-scored weight) at the time of the CPS also is an independent risk factor, the risk increasing with lower z-scored weight. The age at CPS does not influence survival as long as the somatic growth is normal. Although unplanned surgery is associated with poorer outcomes, this is mostly (but not completely) accounted for by the associated risk factors. Interstage reinterventions (with the exception of AVV repair) return patients to predicted risk and could be used to reduce the need for unplanned surgery. Consequently, CPS can be performed safely at an earlier age with no unsatisfactory early or late outcomes as long as these risk factors are absent.

\section{Conflict of Interest Statement}

Authors have nothing to disclose with regard to commercial support.

\section{References}

1. Ohye RG, Sleeper LA, Mahony L, Newburger JW, Pearson GD, Lu M, et al. Comparison of shunt types in the Norwood procedure for single-ventricle lesions. N Engl J Med. 2010;362:1980-92.

2. Ashburn DA, McCrindle BW, Tchervenkov CI, Jacobs ML, Lofland GK, Bove EL, et al. Outcomes after the Norwood operation in neonates with critical aortic stenosis or aortic valve atresia. J Thorac Cardiovasc Surg. 2003;125: 1070-82.

3. Feinstein JA, Benson DW, Dubin AM, Cohen MS, Maxey DM, Mahle WT, et al. Hypoplastic left heart syndrome. Current considerations and expectations. J Am Coll Cardiol. 2012;59:S1-42.

4. Petrucci O, Khoury PR, Manning PB, Eghtesady P. Outcomes of the bidirectional Glenn procedure in patients less than 3 months of age. J Thorac Cardiovasc Surg. 2010;139:562-8.

5. Cnota JF, Allen KR, Colan S, Covitz W, Graham EM, Hehir DA, et al. Superior cavopulmonary anastomosis timing and outcomes in infants with single ventricle. J Thorac Cardiovasc Surg. 2013;145:1288-96.

6. Hehir DA, Dominguez TE, Ballweg JA, Ravishankar C, Marino BS, Bird GL, et al. Risk factors for interstage death after stage 1 reconstruction of hypoplastic left heart syndrome and variants. J Thorac Cardiovasc Surg. 2008; 136:94-9.e1-3.

7. Mahle WT, Spray TL, Gaynor JW, Clark BJ III. Unexpected death after reconstructive surgery for hypoplastic left heart syndrome. Ann Thorac Surg. 2001;71:61-5.

8. Ghanayem N, Tweddell J, Hoffman G, Mussatto K, Jaquiss RDB. Optimal timing of the second stage of palliation for hypoplastic left heart syndrome facilitated through home monitoring, and the results of early cavopulmonary anastomosis. Cardiol Young. 2006;16(Suppl 1):61-6.

9. Chetan D, Kotani Y, Jacques F, Poynter JA, Benson LN, Lee K-J, et al. Surgical palliation strategy does not affect interstage ventricular dysfunction or atrioventricular valve regurgitation in children with hypoplastic left heart syndrome and variants. Circulation. 2013;128(Suppl 1):S205-12.

10. Barron DJ, Brooks A, Stickley J, Woolley SM, Stumper O, Jones TJ, et al. The Norwood procedure using a right ventricle-pulmonary artery conduit: comparison of the right sided verses left sided conduit position. J Thorac Cardiovasc Surg. 2009;138:528-37.

11. Ghanayem NS, Hoffman GM, Mussato KM, Cava JR, Frommelt PC, Rudd NA, et al. Home surveillance program prevents interstage mortality after the Norwood procedure. J Thorac Cardiovasc Surg. 2003;126:1367-75.

12. Hansen JH, Furck AK, Petko C, Buchholz-Berdau R, Voges I, Scheewe J, et al. Use of surveillance criteria reduces interstage mortality after the Norwood procedure for hypoplastic left heart syndrome. Eur J Cardiothorac Surg. 2012;41: 1013-8.

13. Wilder TJ, McCrindle BW, Phillips AB, Blackstone EH, Rajswaran J, Williams WG, et al. Survival and right ventricular performance for matched children after stage-1 Norwood: modified Blalock-Taussig shunt verses right-ventricle-to-pulmonary artery conduit. J Thorac Cardiovasc Surg. 2015; 150:1440-52.

14. Schwarz SM, Lu M, Ohye RG, Hill KD, Atz AM, Naim MY, et al. Risk factors for prolonged length of stay after the stage 2 procedure in the single ventricle reconstruction trial. J Thorac Cardiovasc Surg. 2014;147:1791-8.

15. Hansen JH, Uebing A, Furck AK, Scheewe J, Jung O, Fischer G, et al. Risk factors for adverse outcome after superior cavopulmonary anastomosis for hypoplastic left heart syndrome. Eur J Cardiothorac Surg. 2011;40:e43-9.

16. Tweddell JS, Sleeper LA, Ohye RG, Williams IA, Mahony L, Pizarro C, et al. Intermediate-term mortality and cardiac transplantation in infants with single ventricle lesions: risk factors and their interaction with shunt type. J Thorac Cardiovasc Surg. 2012;144:152-9.

17. McGuirk SP, Stickley J, Griselli M, Stumper OF, Laker SJ, Barron DJ, et al. Risk assessment and early outcome following the Norwood procedure for hypoplastic left heart syndrome. Eur J Cardiothorac Surg. 2006;29:675-81.

18. Newburger JW, Sleeper LA, Frommelt PC, Pearson GD, Mahle WT, Chen S, et al. Transplantation-free survival and interventions at 3 years in the single ventricle trial. Circulation. 2014;129:2013-20.

19. Murtuza B, Stumper O, Wall D, Stickley J, Jones TJ, Barron DJ, et al. The effect of morphological subtype on outcomes following the Sano-Norwood procedure. Eur J Cardiothorac Surg. 2012;42:787-93.

20. Hill KD, Rhodes JF, Aiyagari R, Baker GH, Bergersen L, Chai PJ, et al. Intervention for recoarctation in the single ventricle reconstruction trial: incidence, risk, and outcomes. Circulation. 2013;128:954-61.

21. Jaquiss RD, Ghanayem NS, Hoffman GM, Fedderly RT, Cava JR, Mussatto KA, et al. Early cavopulmonary anastomosis in very young infants after the Norwood procedure: Impact on oxygenation, resource utilization and mortality. J Thorac Cardiovasc Surg. 2004;127:982-9.

22. Kelleher D, Laussen P, Texeira-Pinto A, Duggan C. Growth and correlates of nutritional status among infants with hypoplastic left heart syndrome (HLHS) after stage 1 Norwood procedure. Nutrition. 2006;22: 237-44.

23. Anderson JB, Beekman RH, Border WL, Kalkwarf HJ, Khoury PR, Uzark K, et al. Lower weight-for-age Z-score adversely affects hospital length of stay after the bidirectional Glenn procedure in 100 infants with single ventricle. J Thorac Cardiovasc Surg. 2009;138:397-401.

Key Words: cavopulmonary shunt, Glenn shunt, stage II, Norwood operation, hypoplast left heart syndrome 